\title{
In der Kinder- und Jugendhilfe ist was los!
}

\begin{abstract}
Fünf Jahre Bundeskinderschutzgesetz und fachliche Diskurse rund um die angekündigte SGB-VIII-Reform
Diese Sozial-Extra-Ausgabe widmet sich umfassend vergangenen, aktuellen und zukünftigen Entwicklungen in der Kinder- und Jugendhilfe. Hierbei geht es uns darum, zum kritischen Diskurs anzuregen und dabei sowohl theoretische als auch praxisbezogene Perspektiven aufzuzeigen.
\end{abstract}

$\mathrm{D}$ ie Beiträge in der Rubrik Praxis aktuell beschäftigen sich mit den jüngsten Diskursen um die Reform des SGB VIII und dem fünfjährigen Bestehen des Bundeskinderschutzgesetzes. Hierbei wird in mehreren Beiträgen die vom Bundesministerium initiierte Reform der Kinder- und Jugendhilfe, die unter dem Begriff „Inklusives SGB VIII“ firmiert, und die Fachwelt seit Mitte des letzten Jahres intensiv beschäftigt, aufgegriffen.

\section{Ausgang weiterhin offen}

Obgleich der Ausgang der vom Gesetzgeber angekündigten grundlegenden Reform des SGB VIII - die einen fundamentalen/elementaren Paradigmenwechsel der Kinderund Jugendhilfe bedeuten würde - derzeit offen ist, wird das Thema die Fachwelt auch im Jahr 2017 beschäftigen. Vor diesem Hintergrund wird die Bedeutung der bisher bekannten Zielrichtung der Reform für die Weiterentwicklung der Kinder- und Jugendhilfe diskutiert. Hierzu werden sowohl die Entstehungsgeschichte und die historischen Errungenschaften des SGB VIII als auch aktuelle und per-
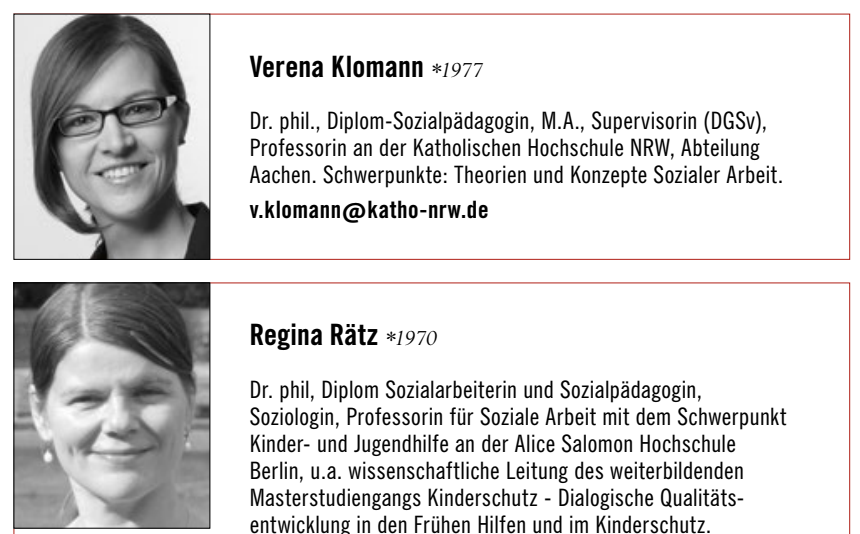

\section{Regina Rätz $* 1970$}

Dr. phil, Diplom Sozialarbeiterin und Sozialpädagogin, Soziologin, Professorin für Soziale Arbeit mit dem Schwerpunkt Kinder- und Jugendhilfe an der Alice Salomon Hochschule Berlin, u.a. wissenschaftliche Leitung des weiterbildenden Masterstudiengangs Kinderschutz - Dialogische Qualitätsentwicklung in den Frühen Hilfen und im Kinderschutz. raetz@ash-berlin.eu spektivische Themen in den Blick genommen sowie Fehlentwicklungen und Alternativen aufgezeigt.

Zudem soll - fünf Jahre nach Inkrafttreten des Bundeskinderschutzgesetzes - deutlich werden, welche Veränderungen in der Praxis angestoßen wurden: Welche Bedeutung und Auswirkungen hatte und hat das ,hochgehandelte Bundeskinderschutzgesetz seit seiner Einführung tatsächlich für die Kinder- und Jugendhilfe? Und: Welche Hoffnungen blieben und bleiben vielleicht unerfüllbar? Zudem werden auf der Basis der Kinder- und Jugendhilfestatistik zentrale und neue Erkenntnisse aufgezeigt, ein Einblick in die Wahrnehmung des Schutzauftrages in den Jugendämtern gewährt und empirische Erkenntnisse zur Bedeutung von Qualifizierungs- und Weiterbildungsangeboten für die Entwicklung von Handlungssicherheit im Kinderschutzkontext erörtert.

\section{Neue Perspektiven}

In den „Durchblick“-Beiträgen werden unter dem Titel „Kinder- und Jugendhilfe: Quo vadis?“ unter Berücksichtigung der bereits angerissenen Entwicklungen und Diskurse um die Neujustierung des SGB VIII grundlegende fachbezogene Themen der Kinder- und Jugendhilfe angesprochen und diskutiert. Diese umfassen Kernthemen der Kinder- und Jugendhilfe wie Inklusion, Partizipation und Kinderrechte. Unter Berücksichtigung bisheriger Erfahrungen sowie gegenwärtiger und zukünftiger Herausforderungen sollen die Themen unter einer weitsichtigen fachlichen Perspektive entwickelt werden. Des Weiteren werden praxisbezogene Erfordernisse zur Inklusion aus der Perspektive von Entwicklungen von jungen Menschen mit Behinderungen diskutiert sowie künftige Veränderungen des Praxisfeldes der Kinder- und Jugendhilfe sowie der Behindertenhilfe angedacht. Hierbei entstehen neue Perspektiven, die über den aktuellen Diskurs zur Reform des SGB VIII hinausgehen und alternative Richtungen aufzeigen. 